\title{
molecules
}

ISSN 1420-3049

www.mdpi.com/journal/molecules

Article

\section{Coupling Two Different Nucleic Acid Circuits in an Enzyme-Free Amplifier}

\author{
Yu Jiang ${ }^{\dagger}$, Bingling $\mathrm{Li}^{\dagger}$, Xi Chen and Andrew D. Ellington * \\ Center for Systems and Synthetic Biology, Department of Chemistry and Biochemistry, \\ Institute for Cellular and Molecular Biology, University of Texas at Austin, Austin, TX 78712, USA \\ $\dagger$ These authors contributed equally to this work. \\ * Author to whom correspondence should be addressed; E-Mail: andy.ellington@mail.utexas.edu; \\ Tel.: +1-512-471-6445; Fax: +1-512-471-7014.
}

Received: 21 September 2012; in revised form: 31 October 2012 / Accepted: 1 November 2012 / Published: 6 November 2012

\begin{abstract}
DNA circuits have proven to be useful amplifiers for diagnostic applications, in part because of their modularity and programmability. In order to determine whether different circuits could be modularly stacked, we used a catalytic hairpin assembly (CHA) circuit to initiate a hybridization chain reaction (HCR) circuit. In response to an input nucleic acid sequence, the CHA reaction accumulates immobilized duplexes and HCR elongates these duplexes. With fluorescein as a reporter each of these processes yielded 10-fold signal amplification in a convenient 96-well format. The modular circuit connections also allowed the output reporter to be readily modified to a G-quadruplexDNAzyme that yielded a fluorescent signal.
\end{abstract}

Keywords: catalyzed hairpin assembly (CHA); hybridization chain reaction (HCR); 96-well plate; enzyme-free

\section{Introduction}

Enzyme-free DNA circuits [1] that rely only on hybridization and strand-exchange reactions to achieve signal amplification have seen increasing use as diagnostic assays. Two of the more robust and programmable DNA circuits are catalytic hairpin assembly (CHA) [2] and the hybridization chain reaction (HCR) [3,4]. CHA and HCR have, respectively, proven useful for amplifying and transducing 
signals from nucleic acid and other analytes into fluorescent, electrochemical, or colorimetric readouts [2,5-7], while HCR has also been used for multiplexed imaging of endogenous mRNA in fixed zebra fish embryos [8].

CHA has proven to be particularly good at staged amplification, while HCR is particularly good at amplification on surfaces such as electrodes or magnetic beads [3,9-11]. Therefore, we hypothesized that a heterogeneous platform that started with CHA amplification and subsequent immobilization of the amplification products might be further amplified by HCR. We were further encouraged by the fact that basic methodologies for heterogeneous platforms (i.e., ELISA assays) have proven extremely successful; for example, the 96-well Neutr-avidin plate used herein has already been widely proofed in assay development $[12,13]$.

In our conception (Scheme 1), a single-stranded nucleic acid analyte $(\mathrm{C} 1)$ can initiate the CHA reaction. The accumulated duplex products $[\mathrm{n}(\mathrm{H} 1: \mathrm{H} 2)]$ have exposed single-stranded DNA tails at the $3^{\prime}$ ends of both $\mathrm{H} 1$ and $\mathrm{H} 2$. The $\mathrm{H} 1$ tail can be further immobilized by hybridization to a Biotin-antisense oligonucleotide on a plate. Following washing, this amplified surrogate of the original nucleic acid analyte provides a substrate for HCR, which can conveniently initiate via the H2 tail (In). The two HCR substrates (H3 and H4) assemble into long concatameric chains [In:(H3:H4)n], and an output signal can be directly read based on the fluorescent labels placed at the 3'ends of H3 and H4.

Scheme 1. Scheme for CHA-HCR two-layer DNA amplifier.

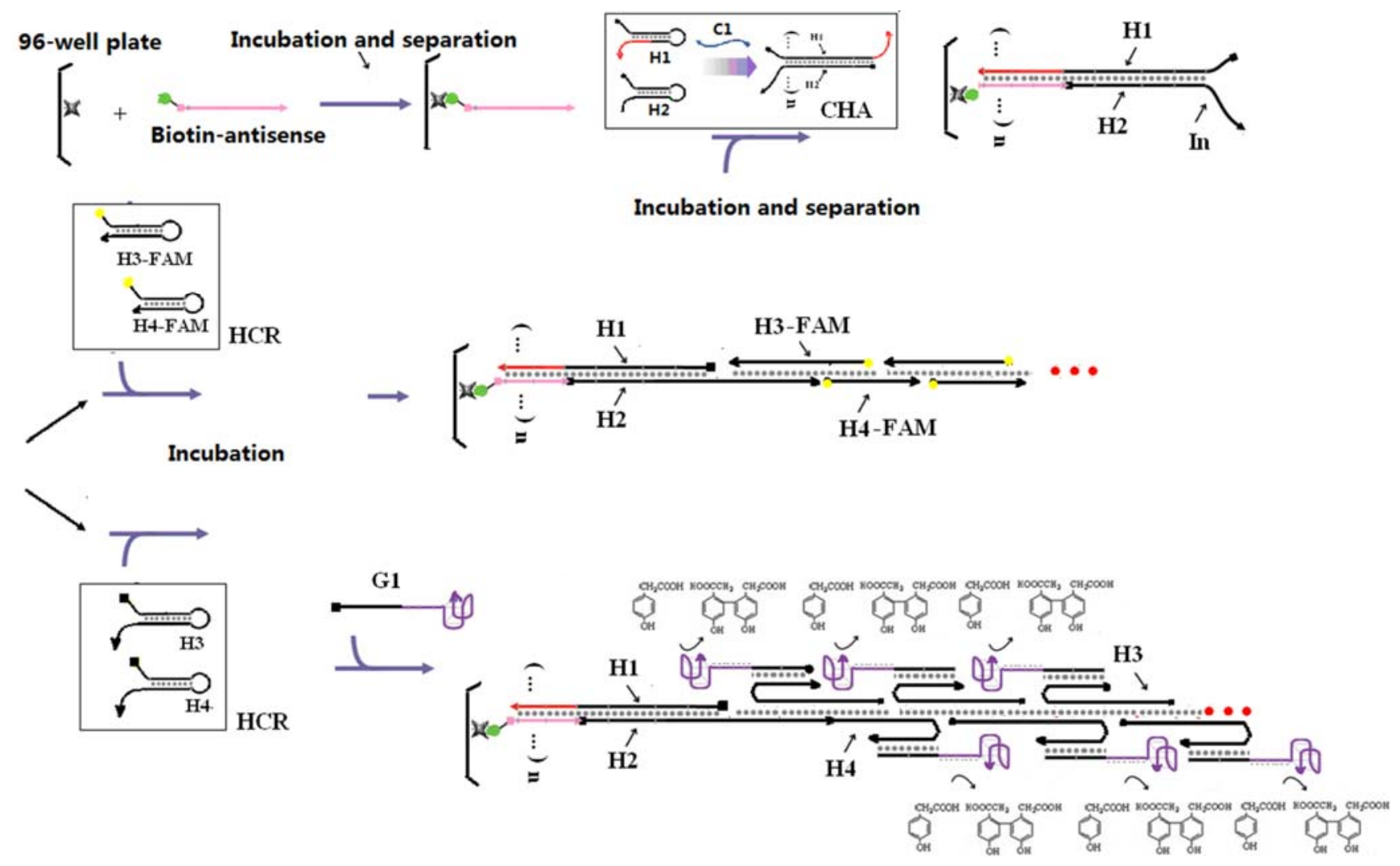

The coupled reaction in fact showed coupled amplification, indicating the relative simplicity of ganging together nucleic acid circuits for diagnostic applications. In order to further demonstrate the versatility of the reaction, we transduced the initial signal into a fluorescent reporter, a G-quantet-hemin DNAzyme. 


\section{Results and Discussion}

\subsection{Designing a CHA-HCR Circuit}

Catalytic hairpin assembly (CHA) and the hybridization chain reaction (HCR) are nucleic acid circuits in which kinetically trapped conformers can react in the presence of a single-stranded sequence-specific trigger or catalyst to form new, more energetically stable conformers. While each reaction starts from two hairpin substrates, CHA yields many duplexes, while HCR yields a double-stranded concatamer.

In order to combine these two circuits, such that CHA would trigger HCR, we first had to position the single-stranded catalyst for HCR on the CHA product (Scheme 2A). The CHA circuit that we used was based on a circuit that had previously shown low background and good amplification [2]. As shown in Scheme 2A, domain 1* (eight residues) on $\mathrm{C} 1$ binds to the toehold domain 1 on the first hairpin substrate $(\mathrm{H} 1)$, initiating a fast branch migration [14] reaction that presents domain $3 *_{-} 4 *$ in a single-stranded conformation. This domain then triggers a second strand displacement with $\mathrm{H} 2$.

Scheme 2. Detailed scheme of CHA (A) and HCR (B) systems.

A

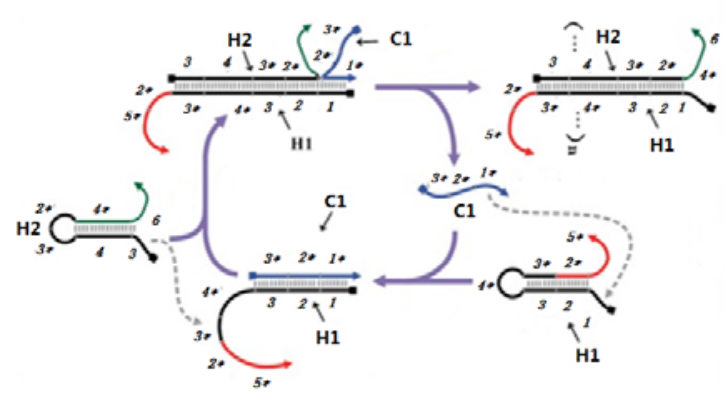

B

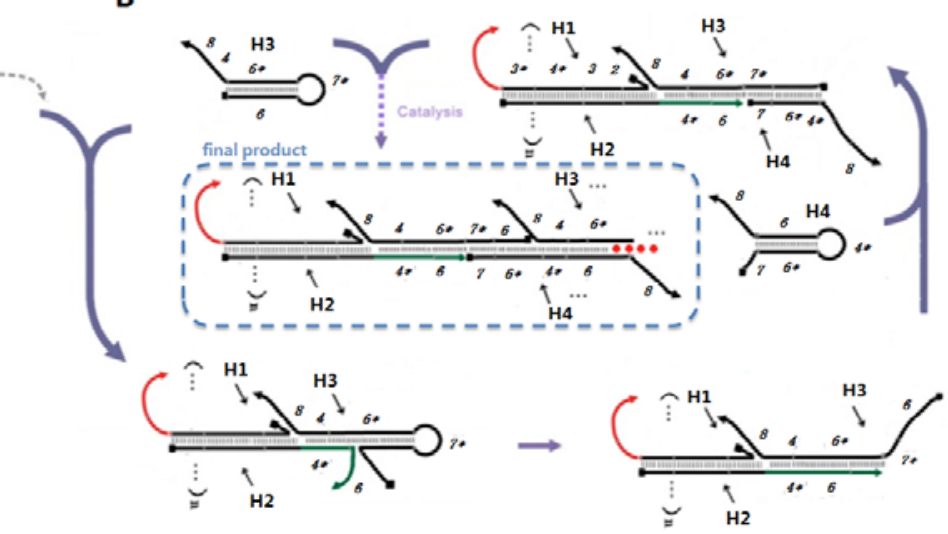

The sequences of the CHA substrates were modified so that the free 3 ' end tails on H1 $(2 *-5 *)$ and H2 (4*-6) could participate in other hybridization and/or strand-exchange reactions. The 16-residue tail on $\mathrm{H} 1$ allows hybridization of the double-stranded CHA product to an antisense sequence to the 16-residue tail (Biotin-antisense) modified streptavidin-coated plates. Since domain $2 *$ is hidden in the initial H1 hairpin, the substrates should not efficiently hybridize to the plates. Single-stranded domain 4*-6 will initiate the HCR.

The HCR circuit was designed based on the principles set out in [4]. The two complementary hairpin substrates $(\mathrm{H} 3, \mathrm{H} 4)$ both contain 18 base-pair stems, six residue loops, and six residue toeholds (Scheme 2B). Upon interaction with domain $4^{*}-6$ of an immobilized H1 with the complementary 4-6* domain on $\mathrm{H} 3$, a strand displacement reaction will lead to opening of $\mathrm{H} 3$ and release of the $7 *-6$ domain, which can in turn hybridize the $7-6^{*}$ domain on $\mathrm{H} 4$, thereby initiating another strand displacement reaction. The newly freed $4 *-6$ domain on $\mathrm{H} 4$ can in turn open a second $\mathrm{H} 3$, followed by iterative opening and reaction of $\mathrm{H} 4, \mathrm{H} 3$, and so forth. As a result of HCR H3 and H4 should join to form an elongated assembly product (as shown in Scheme 2B). Note that compared to the 8-base toehold for the CHA circuit, the HCR toehold had only six residues. Therefore, in order to improve the 
rate of reaction initiation, we used higher salt concentrations $(0.66 \mathrm{M} \mathrm{NaCl})$ and lower temperatures $\left(25^{\circ} \mathrm{C}\right)$ in the HCR portion of the coupled reaction.

The ultimate formation of the HCR product was monitored by including fluorescein at the $3^{\prime}$ ends of $\mathrm{H} 3$ and H4. However, the coupled CHA-HCR cascade should be comparable with a wide range of reporters [10,15-17]. To prove this point, we further replaced the FAM tag on $\mathrm{H} 3$ and $\mathrm{H} 4$ with poly $\mathrm{T}_{18}$ tails that could hybridize to a poly $\mathrm{A}_{18}$-G-quartet-hemin complex. The G-quartet-hemin complex is a wellknown horseradish peroxidase-like DNAzyme that can catalyze the oxidation of p-hydroxyphenylacetic acid (HPA) to the fluorescent product bi-p,p-4-hydroxyphenylacetic acids (Bi-HPA) in the presence of $\mathrm{H}_{2} \mathrm{O}_{2}$. The measured emission at $410 \mathrm{~nm}$ should reflect the presence and concentration of the catalyst strand $\mathrm{C} 1$.

\subsection{Characterization of the CHA and HCR Circuits}

The performance of CHA and HCR circuits was separately verified by native polyacrylamide gel electrophoresis (PAGE), which could reveal what products were being formed as the reaction progressed. For the CHA reaction, after $3 \mathrm{~h}$ of incubation with $10 \mathrm{nM} \mathrm{C1,} 200 \mathrm{nM} \mathrm{H} 1$, and $400 \mathrm{nM} \mathrm{H} 2$, a single high molecular weight product corresponding to $\mathrm{H} 1: \mathrm{H} 2$ appears, while the band corresponding to $\mathrm{H} 1$ almost completely disappears [Figure $1 \mathrm{~A}(\mathrm{~g}))$ ]. While some background duplex formation could be observed even in the absence of $\mathrm{C} 1$ [Figure $1 \mathrm{~A}(\mathrm{f})$ ] the rate of leakage is slow and is likely due to either mis-synthesis and/or mis-folding of $\mathrm{H} 1$ and $\mathrm{H} 2$.

Figure 1. Verification of CHA and HCR products. (A) 15\% PAGE gel after 3-hour CHA reaction. Concentrations of $\mathrm{C} 1, \mathrm{H} 1$, and $\mathrm{H} 2$ (in a to g) are $10 \mathrm{nM}, 200 \mathrm{nM}$, and $400 \mathrm{nM}$, respectively; (B) 8\% PAGE gel after overnight HCR reaction. Concentrations of $\mathrm{H} 3$ and $\mathrm{H} 4$ are both $200 \mathrm{nM}$. Concentrations of the initiator mimic (InM) from a to d are $100 \mathrm{nM}$, $50 \mathrm{nM}, 25 \mathrm{nM}$, and $0 \mathrm{nM}$.
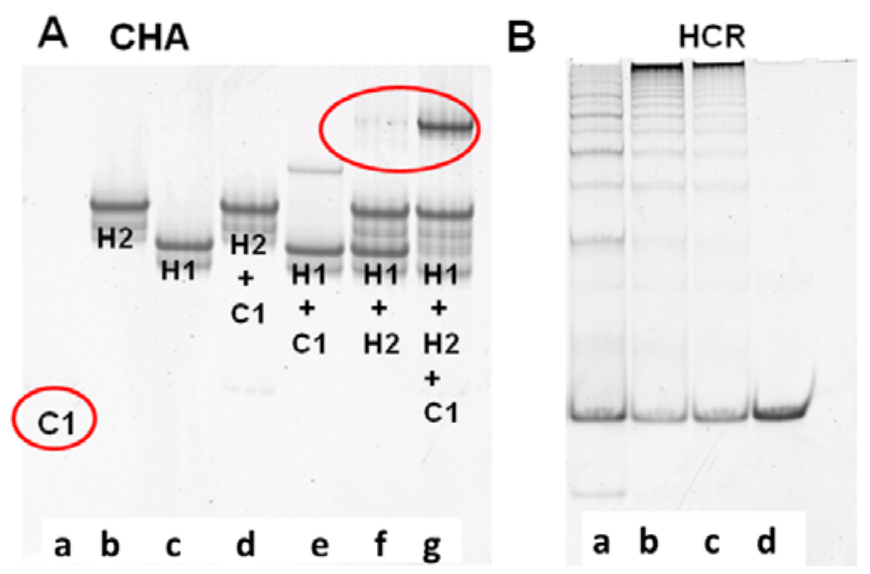

The performance of the CHA circuit could also be confirmed by including an additional substrate, which upon interaction with $\mathrm{H} 1: \mathrm{H} 2$ would lead to strand displacement and release of a FAM-labeled oligonucleotide from a quencher-labeled antisense molecule. As shown in Figure S1, both the C1-dependent catalytic reaction and the background leakage were consistent with the PAGE results (Figure 1A). Since the balance between adequate signal and minimal noise could be most readily observed after a $3 \mathrm{~h}$ reaction, a $3 \mathrm{~h}$ pre-CHA reaction was chosen to initiate the HCR reaction. 
To independently proof the HCR reaction, an initiator mimic (InM, representing the single-stranded $4 *-6$ sequence) was used to initiate the reaction. Figure 1B shows the native gel profile following overnight incubation of $\mathrm{H} 3$ and $\mathrm{H} 4$ with different concentrations of InM. It is obvious that long duplex concatamers (InM:(H3:H4) $)$ are produced in the presence of the InM. Unsurprisingly, the greater the amount of InM the shorter the concatamers produced, since the same amount of substrate is being simultaneously added to multiple, growing HCR chains. The HCR substrates were almost consumed within $5 \mathrm{~h}$ (Figure S1), and the fluorescence of the coupled CHA-HCR cascade could therefore generally be readily monitored after an overnight reaction.

\subsection{Two-Layer Amplification by CHA-HCR}

For assaying analytes, the coupled system was transferred onto microtiter plates. As in Scheme 1, catalyst-dependent CHA in the first stage should produce a sequence-specific bridge to fluorescent HCR products in the second stage. Samples with $\mathrm{C} 1$ indeed produce much higher fluorescence compared with the blank (no $\mathrm{C} 1$; Figure 2A), and the mean fluorescence intensity (calculated from the data points at each $\mathrm{C} 1$ concentration) is concentration-dependent (Figure 2B), with a linear correlation coefficient of 0.94 . As little as $100 \mathrm{pM} \mathrm{C1}$ (three standard deviations above background) could be discriminated from the blank, a limit similar to "on-plate" or "on-fiber" sensors using protein enzymes or nanoparticles for signal amplification [10,15-17].

Figure 2. Kinetics of two-layer amplification by CHA-HCR. (A) Fluorescent kinetic curves after standard CHA-HCR two-layer amplfication; (B) Concentration dependence of $\mathrm{C} 1$ following standard CHA-HCR two-layer amplfication. The RFU for each concentration is the mean of all steady-state data points in Figure 2A.
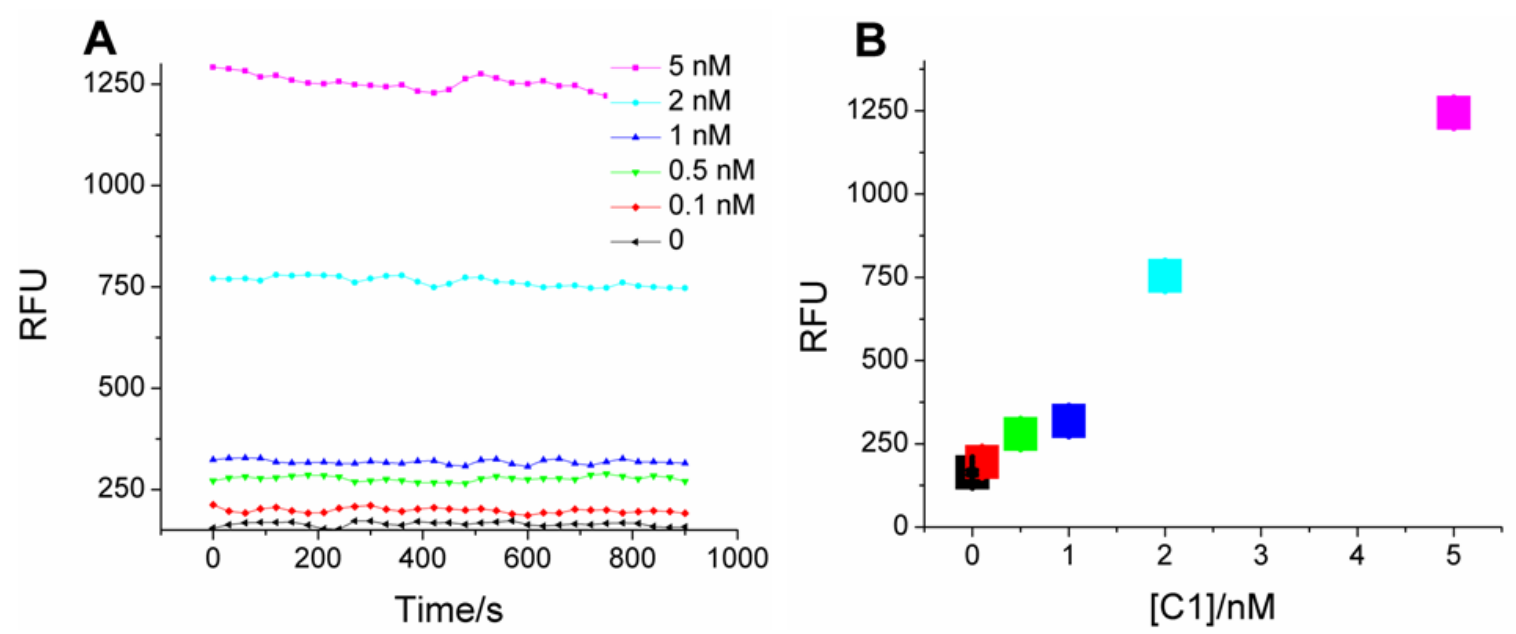

To confirm that both CHA and HCR were contributing to analytical sensitivity, two control experiments are carried out (Figure 3). First, a FAM-labeled complement (FAM-AC) to the immobilized Biotin-antisense was hybridized to mimic a simple DNA sensor without any amplification. Second, FAM-labeled H2 (FAM-H2) was included in the CHA circuit. After triggering by $\mathrm{C} 1$, the CHA products (H1:H2 (FAM)) was hybridized with the Biotin-antisense on plates, to mimic a system with CHA but without HCR amplification. An analyte-dependent fluorescence increase could be observed in both experiments. However, the signal aptitude and sensitivity were 
much less than the standard CHA-HCR amplification (Figure 3, Experiment III). Based on the data in Figures 5, and 6A, it can be calculated that the signal resulting from $5 \mathrm{nM}$ analyte was 10 -fold increased by CHA, and additionally 10-fold increased by HCR, for an overall amplification of ca. 100-fold, confirming two-layer amplification.

Figure 3. Confirmation of the two-layer amplifier. Experiment I: Without any amplification. Experiment II: With only CHA amplifier. Experiment III: With the standard CHA-HCR amplifier. The RFU in each bar graph is the mean of the steady-state data points collected during fluorescence kinetic readings.

Experiment I: Without any amplification
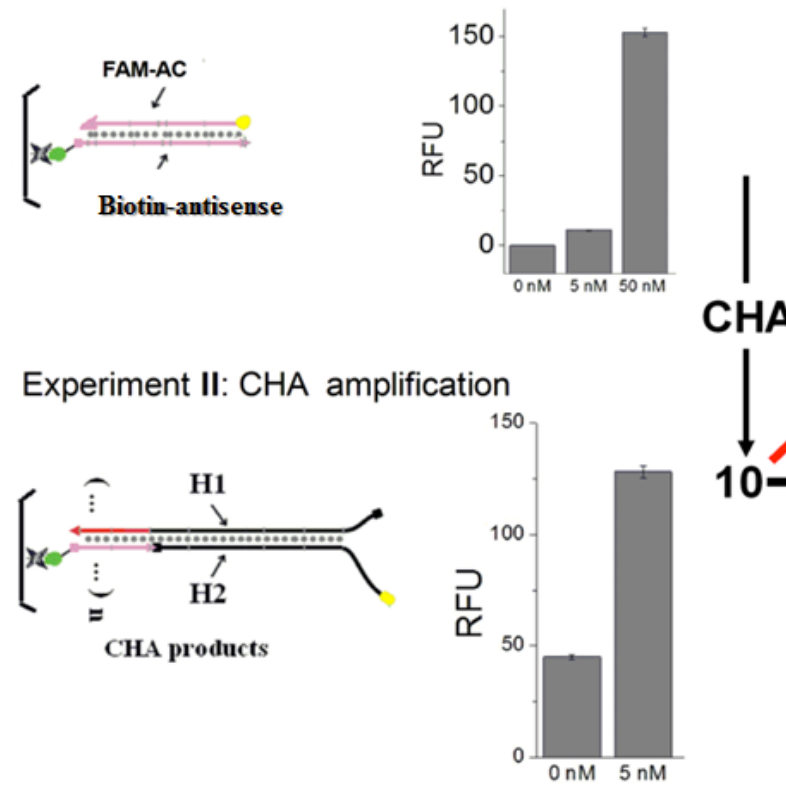

Experiment III: CHA-HCR amplification
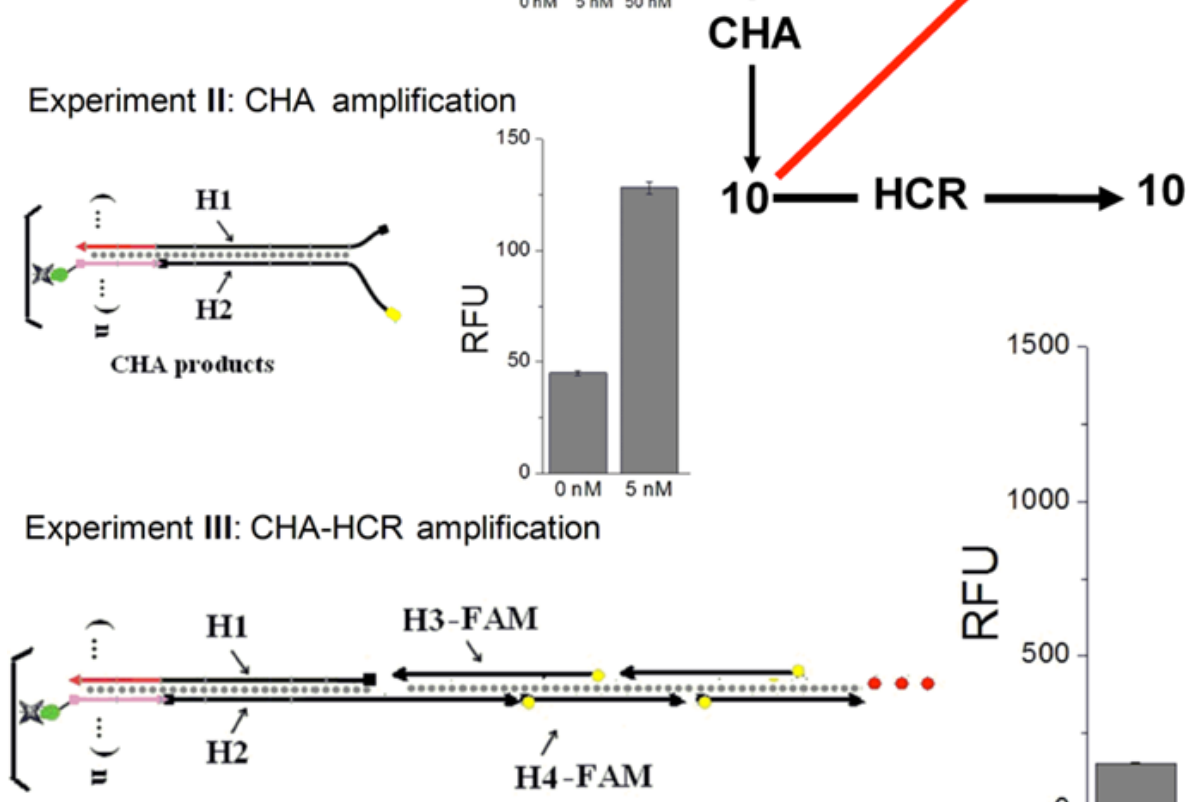

CHA+HCR products

H4-FAM

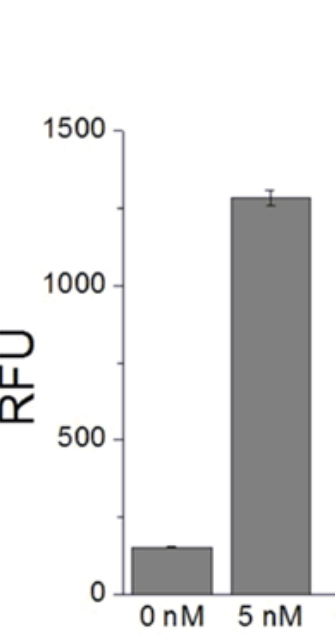

Even greater amplification was limited by the unexpectedly high blank signal (no C1). This signal likely results from CHA leakage, HCR leakage, and non-specific binding to the plate.

\subsection{G-quartet-hemin DNAzyme as a Reporter}

As well known, some G-quartets can bind hemin and thereby form a peroxidase DNAzyme [18,19] G-quartet-hemin DNAzymes catalyze a series of colorimetric and luminous oxidization reactions in the presence of $\mathrm{H}_{2} \mathrm{O}_{2}$, and have been widely used as protein-free signal amplifiers and reporters in biosensors $[18,19]$. We therefore decided to test whether the DNAzyme might provide additional amplification in our coupled CHA-RCA assay. A fluorescent peroxidase substrate (HPA) was chosen and its turnover was found to be dependent upon both $\mathrm{pH}$ and the sequence of the G-quartet. The G-quartets ' $\mathrm{G} 1$ ' and a $\mathrm{pH}$ of 8.5 were chosen for sensing reactions (Figure S2). 
In order to utilize the DNAzyme with the HCR product, the FAM tags on $\mathrm{H} 3$ and $\mathrm{H} 4$ were replaced with poly $\mathrm{T}_{18}$ tails that could hybridize to a polyA ${ }_{18}-\mathrm{G} 1$-hemin DANzyme (Scheme 1). Following the coupled reaction, a C1-dependent fluorescent signal could be observed in the presence of HPA (Figure 4). At the previous limit of detection (100 pM), the DNAzyme gave a 10-fold higher signal amplitude than the FAM reporter. At higher (saturating) concentrations of C1 (5 nM) the DNAzyme still gave a 2-fold higher fluorescent response relative to the FAM reporter. As before, accumulation of background limits sensitivity and signal. It should be noted that though as little as $5 \mathrm{pM}$ of analyte could be reproducibly detected (three standard deviations above background), the DNAzyme reporter did not show a linear dose-response curve (Figure 4B, Figure S3), again in part a consequence of the aforementioned background.

Figure 4. G-quartet-hemin DNAzyme as a reporter for CHA-HCR. (A) Steady-state signal comparison between no amplification, CHA amplification, standard CHA-HCR two-layer amplification, and CHA-HCR-DNAzyme amplification. The RFU is the mean of the steady-state data points collected during fluorescence kinetic readings; (B) Fluorescent kinetic measurement with CHA-HCR-DNAzyme amplification.

A

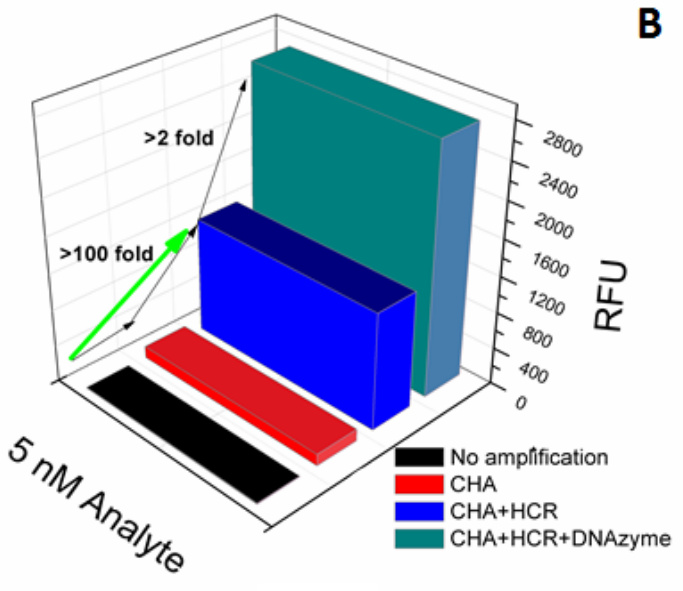

B

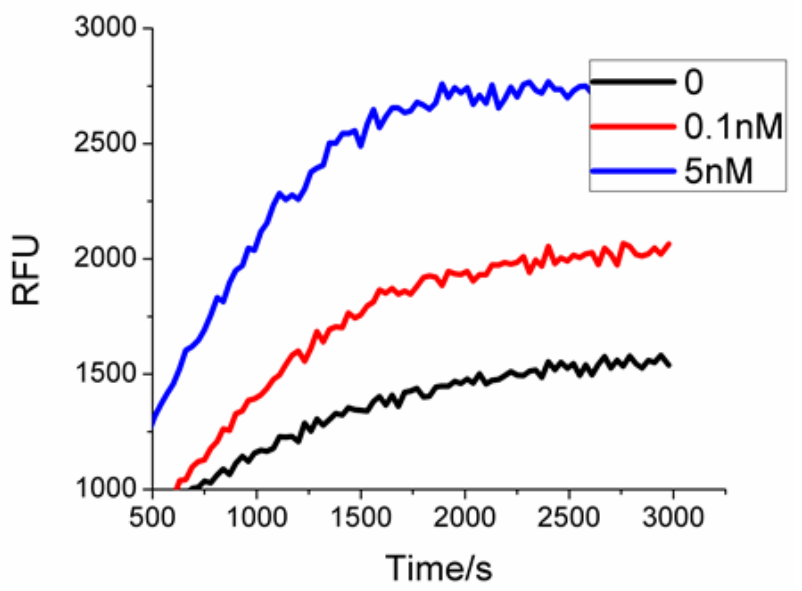

\section{Experimental}

\subsection{Chemicals and Oligonucleotides}

All chemicals were of analytical grade and were purchased from Sigma-Aldrich (St. Louis, MO, USA) unless otherwise indicated. All oligonucleotides were ordered from Integrated DNA Technologies (IDT, Coralville, IA, USA). Oligonucleotide sequences are summarized in Supplementary Table S1. All DNA hairpins (including H1, H2, H3, and H4) were purified via denaturing PAGE (7 M Urea, $1 \times \mathrm{TBE})$. All DNAs were stored in $1 \times \mathrm{TE}(\mathrm{pH} 7.5)$ at a concentration of $1 \mathrm{mM}$. Pierce NeutrAvidin Coated High Capacity Plates (Black, 96-Well) were purchased from Thermo Scientific (Waltham, MA, USA). The wash buffer for the plate was Tris-buffered saline ( $25 \mathrm{mM}$ Tris, $150 \mathrm{mM} \mathrm{NaCl} ; \mathrm{pH} 7.2$ ), $0.1 \%$ BSA, and $0.05 \%$ Tween ${ }^{\circledR}-20$ detergent. 


\subsection{CHA Reactions on the Plate Surface}

Each well was washed three times with $200 \mu \mathrm{L}$ of wash buffer. Some $100 \mu \mathrm{L}$ of $1 \mu \mathrm{M}$ Biotin-antisense in $1 \times \mathrm{TNaK}(20 \mathrm{mM}$ Tris- $\mathrm{HCl}, 140 \mathrm{mM} \mathrm{NaCl}, 5 \mathrm{mM} \mathrm{KCl}, \mathrm{pH} 7.5)$ was added, and the immobilization reaction was incubated at $37{ }^{\circ} \mathrm{C}$ for $2 \mathrm{~h}$. The hairpins $\mathrm{H} 1$ and $\mathrm{H} 2$ were heated to $90{ }^{\circ} \mathrm{C}$ for $5 \mathrm{~min}$ followed by slowly decreasing the temperature to $25{ }^{\circ} \mathrm{C}$ at a rate of $0.1{ }^{\circ} \mathrm{C} / \mathrm{s}$ in order to ensure proper folding. Folded hairpins were incubated with different concentrations of $\mathrm{C} 1$ at $37{ }^{\circ} \mathrm{C}$ for $1.5 \mathrm{~h}$. Each well was washed three times with $200 \mu \mathrm{L}$ wash buffer before adding the CHA reaction. The CHA products then bound to the Biotin-antisense following incubation at $37^{\circ} \mathrm{C}$ for another $1.5 \mathrm{~h}$. Unreacted oligonucleotides were removed by gently washing the plates three times with $200 \mu \mathrm{L}$ wash buffer, and the HCR reaction was initiated by adding $100 \mu \mathrm{L}$ of $100 \mu \mathrm{M} \mathrm{H} 3$ and H4 (the HCR system). The plate was kept at $25^{\circ} \mathrm{C}$ overnight before measurement or further reaction.

\subsection{G-quadruplex with Hemin Catalysis System}

A G-quadruplex that could hybridize to the single-stranded tails of $\mathrm{H} 3$ and $\mathrm{H} 4$ was added to wells where HCR was carried out. After adding $100 \mu \mathrm{L} 100 \mu \mathrm{M}$ of a previously utilized G-quartet, EAD2 (G1) [20] and incubating for $1 \mathrm{~h}$, the plate was washed $200 \mu \mathrm{L}$ wash buffer for three times and then $100 \mu \mathrm{L}$ HPA buffer ( $2 \mathrm{mM}$ HPA; 0.003\% $\mathrm{H}_{2} \mathrm{O}_{2} ; 30 \mathrm{nM}$ Hemin; 0.05\% Triton; $200 \mathrm{mM}$ Tris, pH 8.5; $200 \mathrm{mM} \mathrm{NaCl} ; 200 \mathrm{mM} \mathrm{KCl})$ was added. The plate was then transferred to a TECAN Safire plate reader for fluorescence measurements with an excitation wavelength of $310 \mathrm{~nm}$ and emission wavelength of $410 \mathrm{~nm}$.

\section{Conclusions}

In conclusion, we successfully built a multi-amplifier that utilized three different nucleic acid components: hairpins that executed CHA, hairpins that executed HCR, and G-quadruplex peroxidases. The primary achievement is that nucleic acid circuits can be readily mixed and matched due to the extraordinary programmability provided by base-pairing interactions. However, these results also demonstrated some of the current limitations of nucleic acid conformational transduction as an amplifier mechanism. Because of either false triggering due to the presence of lesions in the hairpins or because of thermal equilibration of the hairpin structures, the background for each layer was relatively high, and the overall amplification seen was relatively low (100-fold comparable to no amplification). While a low limit of detection could be achieved, quantitation proved difficult.

The chief advantage of this analytical method is its simplicity and compatibility with surface immobilization. Unlike polymerase-based reactions, which must generally be constructed from multiple components in order to avoid the degradation of reagents or the production of false amplicons, the nucleic acid hairpins are immediately ready for use and must merely be added to an existing sample or surface. Coupled CHA-HCR circuitry can therefore potentially be adapted for the sensitive 'yes/no' detection of nucleic acid analytes captured from lysates in point-of-care settings. Such circuitry may also prove to be an interesting intermediate for the ligand-dependent immobilization of nucleic acid-enzyme conjugates, providing basal amplification for ELISA-like assays. 


\section{Supplementary Materials}

Supplementary materials can be accessed at: http:/www.mdpi.com/1420-3049/17/11/13211/s1.

\section{Acknowledgments}

This article was funded by National Institute of Health (NIH 1R01EB0007689), the National Security Science and Engineering Faculty Fellowship (NSSEFF FA9550-10-1-0169), Defense Advanced Research Projects Agency (DARPA 5-35830), National Institute of Health (NIH-TR01 5R01AI092839-02) as well as Welch Foundation (F-1654). The published material represents the position of the author(s) and not necessarily that of the sponsors.

\section{References}

1. Zhang, D.Y.; Turberfield, A.J.; Yurke, B.; Winfree, E. Engineering entropy-driven reactions and networks catalyzed by DNA. Science 2007, 318, 1121-1125.

2. Li, B.; Ellington, A.D.; Chen, X. Rational, modular adaptation of enzyme-free DNA circuits to multiple detection methods. Nucleic Acids Res. 2011, 39, e110.

3. Niu, S.; Jiang, Y.; Zhang, S. Fluorescence detection for DNA using hybridization chain reaction with enzyme-amplification. Chem. Commun. 2010, 46, 3089-3091.

4. Dirks, R.M.; Pierce, N.A. Triggered amplification by hybridization chain reaction. Proc. Natl. Acad. Sci. USA 2004, 101, 15275-15278.

5. Choi, H.M.; Chang, J.Y.; Trinhle, A.; Padilla, J.E.; Fraser, S.E.; Pierce, N.A. Programmable in situ amplification for multiplexed imaging of mRNA expression. Nat. Biotechnol. 2010, 28, 1208-1212.

6. Shimron, S.; Wang, F.; Orbach, R.; Willner, I. Amplified detection of DNA through the enzyme-free autonomous assembly of hemin/ G-Quadruplex DNAzyme nanowires. Anal. Chem. 2012, 84, 1042-1048.

7. Zheng, A.; Li, J.; Wang, J.; Song, X.; Chen, G.; Yang, H. Enzyme-free signal amplification in the DNAzyme sensor via target-catalyzed hairpin assembly. Chem. Commun. 2012, 48, 3112-3114.

8. Wang, F.; Elbaz, J.; Orbach, R.; Magen, N.; Willner, I. Amplified analysis of DNA by the autonomous assembly of polymers consisting of DNAzyme wires. J. Am. Chem. Soc. 2011, 133, 17149-17151.

9. Li, B.; Jiang, Y.; Chen, X.; Ellington, A.D. Probing spatial organization of DNA strands using enzyme-free hairpin assembly circuits. J. Am. Chem. Soc. 2012, 134, 13918-13921.

10. Niu, S.; Li, Q.; Ren, R.; Zhang, S. Enzyme-enhanced fluorescence detection of DNA on etched optical fibers. Biosens. Bioelectron. 2009, 24, 2943-2946.

11. Chen, Y.; Xu, J.; Su, J.; Xiang, Y.; Yuan, R.; Chai, Y. In situ hybridization chain reaction amplification for universal and highly sensitive electrochemiluminescent detection of DNA. Anal. Chem. 2012, 84, 7750-7755.

12. Fu, J.; Cai, K.; Johnston, S.A.; Woodbury, N.W. Exploring peptide space for enzyme modulators. J. Am. Chem. Soc. 2010, 132, 6419-6424. 
13. Mishra, N.N.; Pedersen, J.A.; Rogers, K.R. Highly Sensitive Assay for Anticholinesyerase Compounds Using 96 Well Plate Format. In Chemicals in the Environment; Phillips, M.L., Ed.; American Chemical Society: Washington, DC, USA, 2002; Volume 806, Chapter 16, pp. 289-305.

14. Zhang, D.Y.; Winfree, E. Control of DNA strand displacement kinetics using toehold exchange. J. Am. Chem. Soc. 2009, 131, 17303-17314.

15. Ribble, D.; Goldstein, N.B.; Norris, D.A.; Shellman, Y.G. A simple technique for quantifying apoptosis in 96-well plates. BMC Biotechnol. 2005, 5, doi:10.1186/1472-6750-5-12.

16. Mao, X.; Jiang, J.; Xu, X.; Chu, X.; Luo, Y.; Shen, G.; Yu, R. Enzymatic amplification detection of DNA based on "molecular beacon" biosensors. Biosensor 2008, 23, 1555-1561.

17. Niu, Y.; Zhao, Y.; Fan, A. Conformational switching immobilized hairpin DNA probes following subsequent expanding of gold nanoparticles enables visual detecting sequence-specific DNA. Anal. Chem. 2011, 83, 7500-7506.

18. Liu, J.; Cao, Z.; Lu, Y. Functional nucleic acid sensors. Chem. Rev. 2009, 109, 1948-1998.

19. Willner, I.; Shlyahovsky, B.; Zayats, M.; Willner, B. DNAzymes for sensing, nanobiotechnology and logic gate applications. Chem. Soc. Rev. 2008, 37, 1153-1165.

20. Cheng, X.; Liu, X.; Bing, T.; Gao, Z.; Shangguan, D. General peroxidase activity of G-quadruplex-hemin complexes and its application in ligand screening. Biochemistry 2009, 48, 7817-7823.

Sample Availability: Not available.

(C) 2012 by the authors; licensee MDPI, Basel, Switzerland. This article is an open access article distributed under the terms and conditions of the Creative Commons Attribution license (http://creativecommons.org/licenses/by/3.0/). 